\section{Why Has the Ability to Regenerate Following CNS Injury Been Repeatedly Lost Over the Course of Evolution?}

\author{
Seth Blackshaw $1,2,3,4,5 *$ \\ ${ }^{1}$ The Solomon H. Snyder Department of Neuroscience, Johns Hopkins University School of Medicine, Baltimore, MD, \\ United States, ${ }^{2}$ Department of Ophthalmology, Johns Hopkins University School of Medicine, Baltimore, MD, United States, \\ ${ }^{3}$ Department of Neurology, Johns Hopkins University School of Medicine, Baltimore, MD, United States, ${ }^{4}$ Institute for Cell \\ Engineering, Johns Hopkins University School of Medicine, Baltimore, MD, United States, ${ }^{5}$ Kavli Neuroscience Discovery \\ Institute, Johns Hopkins University School of Medicine, Baltimore, MD, United States
}

While many vertebrates can regenerate both damaged neurons and severed axons in the central nervous system (CNS) following injury, others, including all birds and mammals, have lost this ability for reasons that are still unclear. The repeated evolutionary loss of regenerative competence seems counterintuitive, and any explanation must account for the fact that regenerative competence is lost in both cold-blooded and all warm-blooded clades, that both injury-induced neurogenesis and axonal regeneration tend to be lost in tandem, and that mammals have evolved dedicated gene regulatory networks to inhibit injury-induced glia-to-neuron reprogramming. Here, different hypotheses that have been proposed to account for evolutionary loss of regenerative competence are discussed in the light of new insights obtained into molecular mechanisms that control regeneration in the central nervous system. These include pleiotropic effects of continuous growth, enhanced thyroid hormone signaling, prevention of neoplasia, and improved memory consolidation. Recent evidence suggests that the most compelling hypothesis, however, may be selection for greater resistance to the spread of intra-CNS infections, which has led to both enhanced reactive gliosis and a loss of injury-induced neurogenesis and axonal regeneration. Means of testing these hypotheses, and additional data that are urgently needed to better understand the evolutionary pressures and mechanisms driving loss of regenerative competence, are also discussed.

Keywords: regeneration, neurogenesis, vertebrate, mammal, zebrafish, reactive gliosis, infectous disease, axon

\section{THE EVOLUTIONARY LOSS OF INJURY-INDUCED REGENERATIVE COMPETENCE IN CENTRAL NERVOUS SYSTEM}

Most fish and amphibian species can efficiently regenerate damaged neurons and axons in the brain, spinal cord, and/or retina following injury. Regeneration can be robust, rapid, and reproducible: zebrafish, for instance, are able to completely replace retinal photoreceptors lost following light damage more than six times without any clear behavioral defects or morphological abnormalities 
(Ranski et al., 2018). Neuronal regeneration typically occurs by dedifferentiation of glial cells into neuronal progenitors, which then generate new neurons (Tsonis and Del RioTsonis, 2004; Lust and Tanaka, 2019). In salamanders, neuronal regeneration can also occur through dedifferentiation of ependymal cells or transdifferentiation of retinal pigment epithelium (Lust and Tanaka, 2019).

While regenerative competence likely represents an ancestral state for the vast majority of vertebrate species, injury-induced neurogenesis and axonal regeneration have been repeatedly and independently reduced or lost over the course of evolution. For instance, regenerative competence is greatly reduced or lost entirely in the brain and retina of certain fish and amphibians, including medaka (Lust and Wittbrodt, 2018; Shimizu and Kawasaki, 2021) and the clawed frog Xenopus tropicalis (Langhe et al., 2017). Since regenerative competence has been carefully assayed in only a small number of species (Lust and Tanaka, 2019), this is likely to also be true for many other teleost and amphibian species. Furthermore, regenerative competence is far more limited in amniotes. Injury-induced regeneration in adult reptiles is restricted to a few CNS regions, including the forebrain and the tail spinal cord (Duffy et al., 1990; Font et al., 2001). Injury-induced neurogenesis is observed in juvenile (but not adult) chick retina (Fischer and Reh, 2001), and also in the forebrain of adult ring doves, although mammals essentially lack regenerative competence altogether. Regenerative competence in the CNS usually declines with age, sometimes dramatically so (Tanaka and Ferretti, 2009; Yun, 2015; Alunni and Bally-Cuif, 2016). Finally, injury-induced regeneration in the CNS is often but not always correlated with competence to regenerate non-neuronal tissues such as cardiac muscle (Tanaka and Ferretti, 2009; Alunni and Bally-Cuif, 2016; Alesci et al., 2021).

This evolutionary loss of regenerative competence is puzzling. Why would such a seemingly useful ability be independently discarded in multiple evolutionary lineages? Is regeneration following CNS injury actually disadvantageous for many species, or does this reflect an unavoidable trade-off for some other beneficial trait, and if so, what? There are several facts that need to be accounted for in any explanation of this phenomenon. First, while regenerative competence seems to be an evolutionary basal state, it has been lost in many species (Alunni and Bally-Cuif, 2016). Second, loss of competence to regenerate both destroyed neurons and severed axons usually go hand in hand (Ferretti and Géraudie, 1998; Curcio and Bradke, 2018). Third, while glial cells in regenerative species transition rapidly through a reactive-like state before reprogramming into neurogenic progenitors, glial cells in regeneration-incompetent clades such as mammals instead arrest in this state, massively prolonging the duration of reactive gliosis, and often also showing scarring and fibrosis (Burda and Sofroniew, 2014; Escartin et al., 2021). Lastly, recent work (Hoang et al., 2020) shows that dedicated gene regulatory networks inhibit injury-induced glial reprogramming. Here, I critically evaluate explanations that have been proposed to account for the evolutionarily loss of regenerative competence in the light of these observations, discuss experimental approaches to investigate these hypotheses, and highlight other unresolved questions in the field.

\section{PROPOSED EXPLANATIONS FOR EVOLUTIONARY LOSS OF CENTRAL NERVOUS SYSTEM REGENERATIVE COMPETENCE}

\section{Hypothesis 1: Regenerative Competence Is Lost as the Indirect Result of Loss of Continuous Tissue Growth}

Both the body and brain of most cold-blooded vertebrates continue to grow throughout life, and add neurons at all points along the neuraxis to compensate. This has led to the proposal that regenerative competence in adult CNS both arises, and is indirectly lost, in response to adaptations that allow or prevent continuous tissue growth, and is dependent on the presence of dedicated neuronal progenitors that remain active in the CNS throughout life (Holder and Clarke, 1988; Tanaka and Ferretti, 2009). This model makes several testable predictions. First, regenerative competence should correlate directly with ongoing adult neurogenesis. Second, newly generated neurons should typically arise from the same resident neuronal progenitors that contribute to constitutive neurogenesis.

In many cases, patterns of injury-induced neurogenesis do indeed correlate closely with patterns of constitutive neurogenesis, such as in the forebrain of non-mammalian vertebrates (Tanaka and Ferretti, 2009; Grandel and Brand, 2013; Alesci et al., 2021). Injury-induced neurogenesis almost invariably occurs in species and/or CNS regions where continuous neurogenesis is present, implying that ongoing constitutive neurogenesis may be necessary for injury-induced neurogenesis to occur. Furthermore, in some cases, neurons generated as the result of continuous and injury-induced neurogenesis clearly arise from the same progenitor cell population. This is dramatically illustrated by the example of the zebrafish cerebellum, where granule cells are generated throughout life by fate-restricted progenitors, but are the only neuronal cell type regenerated following injury in adulthood (Kaslin et al., 2013, 2017).

In other cases, however, the correlation between ongoing adult neurogenesis and regenerative competence is not precise, with injury-induced neurogenesis often absent where ongoing neurogenesis is present. For instance, the teleost medaka, which shows only highly limited regenerative competence, displays both continuous growth and ongoing neurogenesis (Lust and Wittbrodt, 2018; Shimizu and Kawasaki, 2021). Conversely, while a few discrete mouse brain regions, such as the hippocampal dentate gyrus, show ongoing neurogenesis well into adulthood (Ming and Song, 2011), cells in this region show only limited ability to regenerate damaged neurons following injury, and no evidence for either injury-induced reprogramming of glia or axonal regeneration (Alunni and Bally-Cuif, 2016). These imply that regenerative competence is not necessarily correlated with levels or patterns of ongoing adult neurogenesis. 
Furthermore, neurons generated in response to injury can in some cases arise from different progenitor populations than those used in ongoing adult neurogenesis. The teleost retina contains two populations of resident neurogenic neural progenitors: the ciliary margin zone at the retinal periphery which can generate any neuronal subtype, and a radial gliallike population found throughout the retina that is limited to generating rods (Stenkamp, 2007). Injury-induced neurogenesis, however, is generally thought to occur through reprogramming of a third population-quiescent resident Müller glial cells-to a neural progenitor-like state (Gorsuch and Hyde, 2014; Wan and Goldman, 2016; Lahne et al., 2020), although there remains some controversy as to whether rod-restricted progenitors are truly distinct from the broader population of Müller glia (Stenkamp, 2011).

Lastly, this model does not account for the loss of competence to regenerate severed axons, the extended period of reactive gliosis seen following injury in regeneration-incompetent species, or the active repression of neurogenic competence seen in mammals. On balance, the loss of regenerative competence is highly unlikely to simply occur as an indirect result of the loss of continuous neurogenesis, although this may be so in some cases. Directly testing the model would require the ability to selectively disrupt ongoing neurogenesis without also affecting competence for injury-induced neurogenesis. Since both constitutive and injury-induced neurogenesis are likely to use many of the same molecular mechanisms, both cell-specific and conditional genetic approaches will be needed to address this (Hans et al., 2021).

\section{Hypothesis 2: Regenerative Competence Is Lost as the Result of Increased Thyroid Hormone Signaling}

Thyroid hormone levels are often inversely correlated with regenerative competence. Thyroid hormone signaling actively inhibits cardiac muscle regeneration (Hirose et al., 2019; Marshall et al., 2019). Thyroid hormone acts as a trigger for amphibian metamorphosis (Brown and Cai, 2007), following which regenerative competence is substantially reduced. Increased thyroid hormone levels also accelerate developmental loss of axonal regeneration in cerebellar Purkinje cells (Avci et al., 2012). This has led to the proposal that CNS regenerative competence may have been indirectly suppressed as the result of selection for increased thyroid hormone signaling (Tanaka and Ferretti, 2009). This model predicts that thyroid hormone signaling will actively inhibit regenerative competence in all vertebrates, and that its disruption should restore regenerative competence in regeneration-incompetent species such as mammals.

Evidence to support this model is limited, however. Although regenerative competence is decreased relative to the larval stage, salamanders and Xenopus laevis retain regenerative competence following metamorphosis (Alunni and Bally-Cuif, 2016; Alesci et al., 2021). Furthermore, thyroid hormone signaling stimulates both adult neurogenesis and functional recovery following injury (Montero-Pedrazuela et al., 2006; Fanibunda et al., 2018). Finally, the effects of manipulating thyroid hormone signaling in regeneration-competent zebrafish are complex. Both dose and context-dependence are observed, with thyroid hormone in some cases stimulating regeneration (Bhumika and Darras, 2014). While thyroid hormone signaling may indeed modulate regenerative competence, its effect is not consistently positive or negative. Nonetheless, regenerative competence has not been carefully tested in either dietary-induced hypothyroidism, nor following genetic disruption of either thyroid hormone synthesis or signaling. It remains formally possible that reductions in thyroid hormone signaling may restore some levels of regenerative competence to mammals.

\section{Hypothesis 3: Loss of Regenerative Competence Is an Adaptation That Promotes Resistance to Cancer}

High levels of ongoing cell proliferation are associated with increased mutation rates and formation of cancers. Since a reduction in proliferation may indirectly inhibit neoplasia, this has been proposed as a mechanism that underlies the loss of regenerative competence in longer-lived species (Tanaka and Ferretti, 2009; Chung, 2018). This model predicts that regeneration-competent species will show higher rates of cancers such as glioblastoma and neuroblastoma-like tumors. In addition, regenerative competence might be expected to decrease with age, as the probability of tumor formation increases (Kitsoulis et al., 2020).

It is difficult to evaluate the accuracy of this hypothesis, as tumor incidence in regeneration-competent vertebrates has generally not been extensively characterized. However, tumor incidence in zebrafish is lower than in mice for both spontaneous and genetically induced tumors (Feitsma and Cuppen, 2008; Hason and Bartůněk, 2019). Furthermore, while tumor incidence rises with age in zebrafish as in mammals (Raby et al., 2020), no clear age-dependent reduction in regenerative competence is observed (Yun, 2015). Cancer rates are also highly variable among regeneration-incompetent mammalian species, and are inversely correlated with reproductive lifespan (Albuquerque et al., 2018). It does not explain why axonal regeneration would be lost along with injury-induced neurogenesis, or why gliosis is extended in regeneration-incompetent species. Overall, this does not appear to be a likely explanation for the evolutionary loss of regenerative competence.

\section{Hypothesis 4: Loss of Regenerative Competence Promotes Improved Memory Consolidation}

Regeneration-induced remodeling of the CNS can, in principle, disrupt pre-existing patterns of neuronal connectivity and synaptic potentiation. In light of the loss of regenerative competence in mammals, it has been proposed that this loss has occurred secondarily to enhanced long-term memory (Kiernan, 1979; Larner et al., 1995). This model predicts that cold-blooded vertebrates with reduced regenerative competence, such as medaka, should show improved long-term memory. Furthermore, neurogenesis and axonal regeneration should disrupt long-term memory consolidation. 


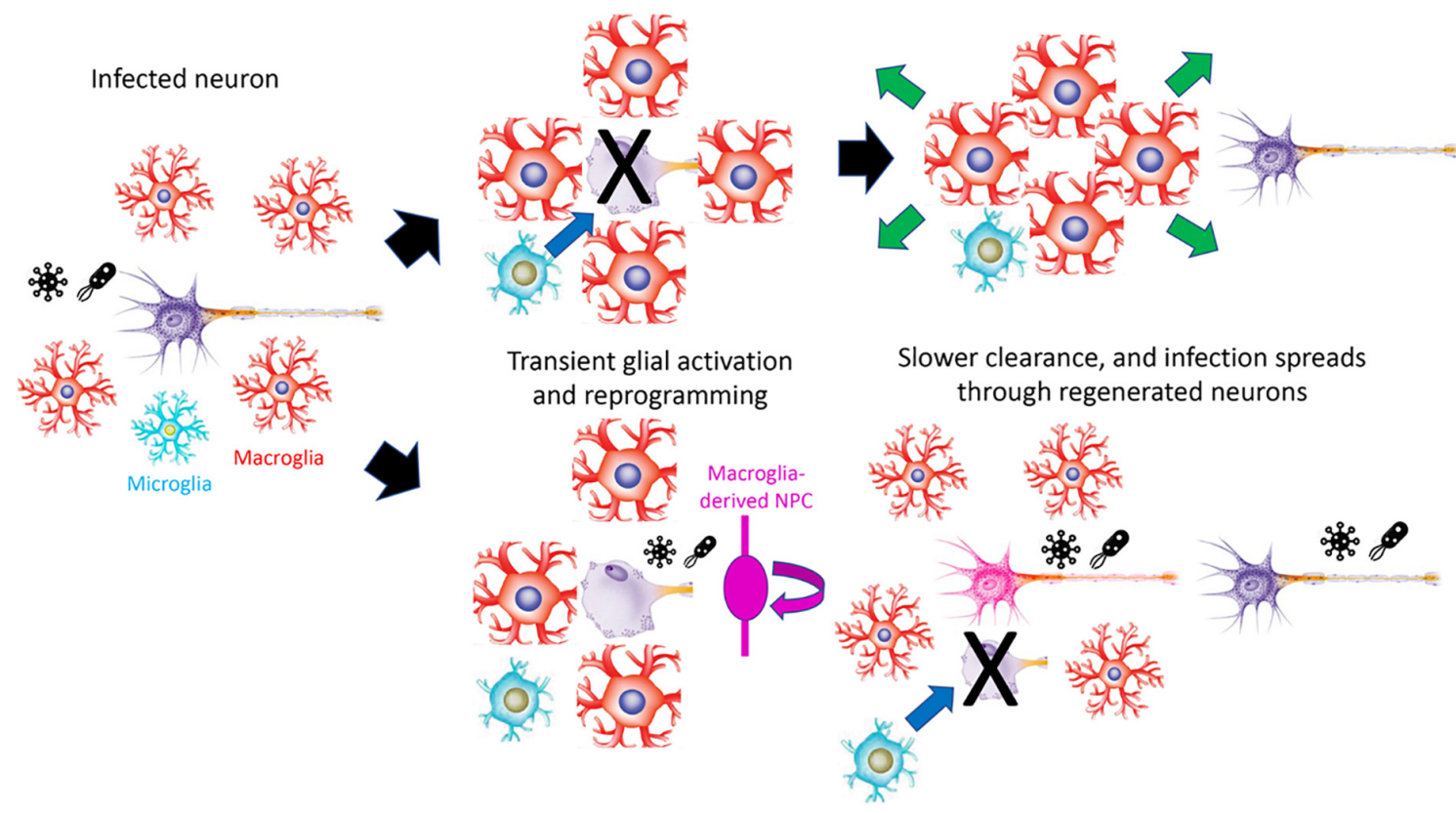

FIGURE 1 | Model for how prolonged reactive gliosis and loss of regenerative competence may protect the CNS against infection. Infection leads to extended reactive gliosis and destruction of infected cells in regeneration-incompetent organisms, restricting the spread of infection. In regeneration-competent organisms, however, limited gliosis and rapid regeneration can lead to intra-CNS spread of infection. For clarity, macroglia are shown as astroglial-like rather than the radial morphology adopted in many regeneration-competent species. NPC, neural progenitor cell.

There is currently no concrete evidence to support this hypothesis, although long-term memory retention following neuronal regeneration has yet to be investigated. Adult neurogenesis in the olfactory bulb and dentate gyrus in mammals has been linked to long-term memory formation, not erasure (Gage, 2019; Tuncdemir et al., 2019). Loss of regenerative competence occurs in both the brain and other CNS regions, such as retina and spinal cord, where experience-dependent plasticity is substantially lower (Tanaka and Ferretti, 2009; Alunni and Bally-Cuif, 2016; Alesci et al., 2021). Likewise, there is no evidence that regeneration-incompetent species show improved long-term memory, although this awaits rigorous investigation. Overall, this too seems an unlikely explanation for loss of regenerative competence.

\section{Hypothesis 5: Loss of Regenerative Competence Increases Resistance to Infection}

Infectious diseases and parasites are a common cause of premature mortality in virtually all species, and resistance to infection is a major selective pressure (Karlsson et al., 2014). Since the CNS is largely isolated from ongoing adaptive immune surveillance (Forrester et al., 2018), the consequences of breakthrough infections are potentially severe. A final hypothesis to explain the evolutionary loss of regenerative competence is that it confers resistance to intra-CNS infection, both by blocking proliferation and axonal outgrowth of infected cells and by enhancing and prolonging reactive gliosis (Rattner and Nathans, 2006; Hoang et al., 2020).

In contrast to the other models proposed, this largely accounts for the observed differences in injury response seen between regeneration-competent and -incompetent organisms. It explains the otherwise mysterious phenomenon of the enhanced and prolonged reactive gliosis observed following injury in mammals (Burda and Sofroniew, 2014). Reactive gliosis consists of three major components. Activated glia first swell and become rigid, forming a physical barrier. Working in tandem with microglia, they then kill and consume damaged cells, and in parallel, they release a cocktail of secreted factors that protect cells not targeted for destruction. Genetic studies have demonstrated that reactive gliosis greatly restricts the spread of intra-CNS pathogens in non-regeneration competent species (Drögemüller et al., 2008; Burda and Sofroniew, 2014). Enhancing and prolonging this process, while also eliminating pathogen spread that is potentially induced by proliferation, neurogenesis and axonal outgrowth, is a potent defense against the spread of infection (Figure 1). While modest levels of inflammation are required to initiate glial activation and reprogramming (Kyritsis et al., 2012; Wan and Goldman, 2016; Bollaerts et al., 2017; Hoang et al., 2020), high and/or sustained levels of inflammation can inhibit both injury-induced neurogenesis and axonal regeneration in the CNS (White et al., 2017; Palazzo et al., 2020). As exposure to intraCNS parasites, and their associated inflammatory responses, can vary dramatically between closely-related organisms that occupy different ecological niches (Scharsack et al., 2007; 
Peuß et al., 2020), this may account for the loss of regenerative competence in some cold-blooded species.

This model makes several predictions. First, inhibiting regeneration and prolonging reactive gliosis should improve resistance to intra-CNS infection in regeneration-competent species. Second, since this proposed mechanism only leads to a loss of injury-induced neurogenesis, a completely separate molecular mechanism is likely to control loss of injuryinduced neurogenesis and axonal regeneration. Third, loss of regenerative competence should directly correlate with increased risk of intra-CNS infection, at least in the recent geological past. This might result from increased parasite load, exposure, and/or pathogenicity.

Testing the first of these predictions is now feasible, using cellspecific, conditional genetic tools and multiomic data available in zebrafish, together with established models of intra-CNS infection (Palha et al., 2013; Passoni et al., 2017; Takaki et al., 2018). Testing the second prediction awaits a thorough analysis of gene regulatory networks controlling the response to axonal injury in both regeneration-competent and incompetent species, as has been done for injury-induced neurogenesis. The third prediction is harder to test, as epidemiological data for wild populations is rarely available and difficult to acquire. However, in some cases, such as surface- and cave-dwelling tetra fish, clear differences in both the parasite load and the response to infection have been described (Peuß et al., 2020), and it should be straightforward to test whether these correlate with differences in regenerative competence.

\section{WHAT DO WE STILL NEED TO KNOW?}

To understand how regenerative competence has been lost over the course of evolution, and to distinguish among these competing hypotheses, additional research is urgently needed. Most importantly, we need a more extensive comparative characterization of regenerative competence. This has been

\section{REFERENCES}

Albuquerque, T. A. F., Drummond do Val, L., Doherty, A., and de Magalhães, J. P. (2018). From humans to hydra: patterns of cancer across the tree of life. Biol. Rev. Camb. Philos. Soc. 93, 1715-1734. doi: 10.1111/brv.12415

Alesci, A., Pergolizzi, S., Lo Cascio, P., Fumia, A., and Lauriano, E. R. (2021). Neuronal regeneration: vertebrates comparative overview and new perspectives for neurodegenerative diseases. Acta Zool. doi: 10.1111/azo.12397

Alunni, A., and Bally-Cuif, L. (2016). A comparative view of regenerative neurogenesis in vertebrates. Development 143, 741-753. doi: 10.1242/dev. 122796

Avci, H. X., Lebrun, C., Wehrlé, R., Doulazmi, M., Chatonnet, F., Morel, M.P., et al. (2012). Thyroid hormone triggers the developmental loss of axonal regenerative capacity via thyroid hormone receptor $\alpha 1$ and krüppel-like factor 9 in Purkinje cells. Proc. Natl. Acad. Sci. U.S.A. 109, 14206-14211. doi: 10.1073/ pnas.1119853109

Bhumika, S., and Darras, V. M. (2014). Role of thyroid hormones in different aspects of nervous system regeneration in vertebrates. Gen. Comp. Endocrinol. 203, 86-94. doi: 10.1016/j.ygcen.2014.03.017

Bollaerts, I., Van Houcke, J., Andries, L., De Groef, L., and Moons, L. (2017). Neuroinflammation as Fuel for Axonal Regeneration in the Injured Vertebrate systematically investigated in only a small number of fish, amphibians, and reptiles, and even more rarely has this analysis been done across different CNS regions in individual species. This will both aid efforts to correlate individual ecological, behavioral, and physiological traits with the loss or retention of regenerative competence and provide additional model systems for molecular analysis. This comparative analysis needs to be extended to comprehensive characterization of injury-activated gene regulatory networks in both neurons and glia, investigating whether general principles obtained from the analysis of zebrafish and mice extend more broadly to regeneration-competent and incompetent organisms, and also determine whether they extend to more broadly regeneration-competent and -incompetent CNS regions within the same species. Finally, given the tantalizing evidence for a link between loss of regenerative competence and resistance to infection, we urgently need a more extensive understanding of how this correlates with overall exposure to pathogens that target the CNS and the resulting immune response, as well as to understand how regeneration-competent organisms respond to these infections (Godwin et al., 2017).

\section{AUTHOR CONTRIBUTIONS}

SB conceived and wrote this manuscript.

\section{FUNDING}

SB is supported by a Stein Innovation Award from Research to Prevent Blindness.

\section{ACKNOWLEDGMENTS}

SB would like to thank J. Wittbrodt, N. Rohner, M. Mommersteeg, J. Nathans, J. Mumm, M. Placzek, W. Yap, and members of the Blackshaw lab for helpful comments.

Central Nervous System. Mediators Inflamm. 2017:9478542. doi: 10.1155/2017/ 9478542

Brown, D. D., and Cai, L. (2007). Amphibian metamorphosis. Dev. Biol. 306, 20-33. doi: 10.1016/j.ydbio.2007.03.021

Burda, J. E., and Sofroniew, M. V. (2014). Reactive gliosis and the multicellular response to CNS damage and disease. Neuron 81, 229-248. doi: 10.1016/j. neuron.2013.12.034

Chung, K.-E. (2018). Comparison of regenerative neurogenesis in response to CNS injury between adult zebrafish and mice. Univ. Ott. Sci. Undergrad. Res. J. 1, 37-41. doi: 10.18192/osurj.v1i1.3696

Curcio, M., and Bradke, F. (2018). Axon Regeneration in the Central Nervous System: facing the Challenges from the Inside. Annu. Rev. Cell Dev. Biol. 34, 495-521. doi: 10.1146/annurev-cellbio-100617-062508

Drögemüller, K., Helmuth, U., Brunn, A., Sakowicz-Burkiewicz, M., Gutmann, D. H., Mueller, W., et al. (2008). Astrocyte gp130 expression is critical for the control of Toxoplasma encephalitis. J. Immunol. 181, 2683-2693. doi: 10.4049/ jimmunol.181.4.2683

Duffy, M. T., Simpson, S. B. Jr., Liebich, D. R., and Davis, B. M. (1990). Origin of spinal cord axons in the lizard regenerated tail: supernormal projections from local spinal neurons. J. Comp. Neurol. 293, 208-222. doi: 10.1002/cne. 902930205 
Escartin, C., Galea, E., Lakatos, A., O’Callaghan, J. P., Petzold, G. C., SerranoPozo, A., et al. (2021). Reactive astrocyte nomenclature, definitions, and future directions. Nat. Neurosci. 24, 312-325. doi: 10.1038/s41593-02000783-4

Fanibunda, S. E., Desouza, L. A., Kapoor, R., Vaidya, R. A., and Vaidya, V. A. (2018). Thyroid Hormone Regulation of Adult Neurogenesis. Vitam. Horm. 106, 211-251. doi: 10.1016/bs.vh.2017.04.006

Feitsma, H., and Cuppen, E. (2008). Zebrafish as a Cancer Model. Mol. Cancer Res. 6, 685-694. doi: 10.1158/1541-7786.mcr-07-2167

Ferretti, P., and Géraudie, J. (1998). Cellular and Molecular Basis of Regeneration: from Invertebrates to Humans. New York, NY: John Wiley \& Sons Incorporated.

Fischer, A. J., and Reh, T. A. (2001). Müller glia are a potential source of neural regeneration in the postnatal chicken retina. Nat. Neurosci. 4, 247-252. doi: $10.1038 / 85090$

Font, E., Desfilis, E., Pérez-Cañellas, M. M., and García-Verdugo, J. M. (2001). Neurogenesis and neuronal regeneration in the adult reptilian brain. Brain Behav. Evol. 58, 276-295. doi: 10.1159/000057570

Forrester, J. V., McMenamin, P. G., and Dando, S. J. (2018). CNS infection and immune privilege. Nat. Rev. Neurosci. 19, 655-671. doi: 10.1038/s41583-0180070-8

Gage, F. H. (2019). Adult neurogenesis in mammals. Science 364, $827-828$.

Godwin, J. W., Pinto, A. R., and Rosenthal, N. A. (2017). Chasing the recipe for a pro-regenerative immune system. Semin. Cell Dev. Biol. 61, 71-79.

Gorsuch, R. A., and Hyde, D. R. (2014). Regulation of Müller glial dependent neuronal regeneration in the damaged adult zebrafish retina. Exp. Eye Res. 123, 131-140. doi: 10.1016/j.exer.2013.07.012

Grandel, H., and Brand, M. (2013). Comparative aspects of adult neural stem cell activity in vertebrates. Dev. Genes Evol. 223, 131-147. doi: 10.1007/s00427-0120425-5

Hans, S., Zöller, D., Hammer, J., Stucke, J., Spieß, S., Kesavan, G., et al. (2021). Cre-Controlled CRISPR mutagenesis provides fast and easy conditional gene inactivation in zebrafish. Nat. Commun. 12:1125. doi: 10.1038/s41467-02121427-6

Hason, M., and Bartůněk, P. (2019). Zebrafish Models of Cancer-New Insights on Modeling Human Cancer in a Non-Mammalian Vertebrate. Genes 10:935. doi: 10.3390/genes10110935

Hirose, K., Payumo, A. Y., Cutie, S., Hoang, A., Zhang, H., Guyot, R., et al. (2019). Evidence for hormonal control of heart regenerative capacity during endothermy acquisition. Science 364, 184-188. doi: 10.1126/science.aar 2038

Hoang, T., Wang, J., Boyd, P., Wang, F., Santiago, C., Jiang, L., et al. (2020). Gene regulatory networks controlling vertebrate retinal regeneration. Science 370:eabb8598. doi: 10.1126/science.abb8598

Holder, N., and Clarke, J. D. W. (1988). Is there a correlation between continuous neurogenesis and directed axon regeneration in the vertebrate nervous system? Trends Neurosci. 11, 94-99. doi: 10.1016/0166-2236(88)90151-8

Karlsson, E. K., Kwiatkowski, D. P., and Sabeti, P. C. (2014). Natural selection and infectious disease in human populations. Nat. Rev. Genet. 15, 379-393. doi: $10.1038 / \mathrm{nrg} 3734$

Kaslin, J., Kroehne, V., Benato, F., Argenton, F., and Brand, M. (2013). Development and specification of cerebellar stem and progenitor cells in zebrafish: from embryo to adult. Neural Dev. 8:9. doi: 10.1186/1749-8104-8-9

Kaslin, J., Kroehne, V., Ganz, J., Hans, S., and Brand, M. (2017). Distinct roles of neuroepithelial-like and radial glia-like progenitor cells in cerebellar regeneration. Development 144, 1462-1471.

Kiernan, J. A. (1979). Hypotheses concerned with axonal regeneration in the mammalian nervous system. Biol. Rev. Camb. Philos. Soc. 54, 155-197. doi: 10.1111/j.1469-185x.1979.tb00871.x

Kitsoulis, C. V., Baxevanis, A. D., and Abatzopoulos, T. J. (2020). The occurrence of cancer in vertebrates: a mini review. J. Biol. Res. 27:9. doi: 10.1186/s40709020-00119-0

Kyritsis, N., Kizil, C., Zocher, S., Kroehne, V., Kaslin, J., Freudenreich, D., et al. (2012). Acute inflammation initiates the regenerative response in the adult zebrafish brain. Science 338, 1353-1356. doi: 10.1126/science.1228773
Lahne, M., Nagashima, M., Hyde, D. R., and Hitchcock, P. F. (2020). Reprogramming Müller Glia to Regenerate Retinal Neurons. Annu. Rev. Vis. Sci. 6, 171-193. doi: 10.1146/annurev-vision-121219-081808

Langhe, R., Chesneau, A., Colozza, G., Hidalgo, M., Ail, D., Locker, M., et al. (2017). Müller glial cell reactivation in Xenopus models of retinal degeneration. Glia 65, 1333-1349. doi: 10.1002/glia.23165

Larner, A. J., Johnson, A. R., and Keynes, R. J. (1995). Regeneration in the vertebrate central nervous system: phylogeny, ontogeny, and mechanisms. Biol. Rev. Camb. Philos. Soc. 70, 597-619. doi: 10.1111/j.1469-185x.1995.tb01653.x

Lust, K., and Tanaka, E. M. (2019). A Comparative Perspective on Brain Regeneration in Amphibians and Teleost Fish. Dev. Neurobiol. 79, 424-436. doi: 10.1002/dneu.22665

Lust, K., and Wittbrodt, J. (2018). Activating the regenerative potential of Müller glia cells in a regeneration-deficient retina. Elife 7:e32319. doi: 10.7554/eLife. 32319

Marshall, L. N., Vivien, C. J., Girardot, F., Péricard, L., Scerbo, P., Palmier, K., et al. (2019). Stage-dependent cardiac regeneration in Xenopus is regulated by thyroid hormone availability. Proc. Nat. Acad. Sci. U.S.A. 116, 3614-3623. doi: $10.1073 /$ pnas. 1803794116

Ming, G.-L., and Song, H. (2011). Adult neurogenesis in the mammalian brain: significant answers and significant questions. Neuron 70, 687-702. doi: 10.1016/ j.neuron.2011.05.001

Montero-Pedrazuela, A., Venero, C., Lavado-Autric, R., Fernández-Lamo, I., García-Verdugo, J. M., Bernal, J., et al. (2006). Modulation of adult hippocampal neurogenesis by thyroid hormones: implications in depressive-like behavior. Mol. Psychiatry 11, 361-371. doi: 10.1038/sj.mp.4001802

Palazzo, I., Deistler, K., Hoang, T. V., Blackshaw, S., and Fischer, A. J. (2020). NF-кB signaling regulates the formation of proliferating Müller glia-derived progenitor cells in the avian retina. Development 147:dev183418. doi: 10.1242/dev.18 3418

Palha, N., Guivel-Benhassine, F., Briolat, V., Lutfalla, G., Sourisseau, M., Ellett, F., et al. (2013). Real-time whole-body visualization of Chikungunya Virus infection and host interferon response in zebrafish. PLoS Pathog. 9:e1003619. doi: 10.1371/journal.ppat.1003619

Passoni, G., Langevin, C., Palha, N., Mounce, B. C., Briolat, V., Affaticati, P., et al. (2017). Imaging of viral neuroinvasion in the zebrafish reveals that Sindbis and chikungunya viruses favour different entry routes. Dis. Model. Mech. 10, 847-857. doi: 10.1242/dmm.029231

Peuß, R., Box, A. C., Chen, S., Wang, Y., Tsuchiya, D., Persons, J. L., et al. (2020). Adaptation to low parasite abundance affects immune investment and immunopathological responses of cavefish. Nat. Ecol. Evol. 4, 1416-1430. doi: 10.1038/s41559-020-1234-2

Raby, L., Völkel, P., Le Bourhis, X., and Angrand, P.-O. (2020). Genetic Engineering of Zebrafish in Cancer Research. Cancers 12:2168. doi: 10.3390/ cancers 12082168

Ranski, A. H., Kramer, A. C., Morgan, G. W., Perez, J. L., and Thummel, R. (2018). Characterization of retinal regeneration in adult zebrafish following multiple rounds of phototoxic lesion. PeerJ 6:e5646. doi: 10.7717/peerj. 5646

Rattner, A., and Nathans, J. (2006). An evolutionary perspective on the photoreceptor damage response. Am. J. Ophthalmol. 141, 558-562. doi: 10. 1016/j.ajo.2005.10.045

Scharsack, J. P., Kalbe, M., Harrod, C., and Rauch, G. (2007). Habitat-specific adaptation of immune responses of stickleback (Gasterosteus aculeatus) lake and river ecotypes. Proc. Biol. Sci. 274, 1523-1532. doi: 10.1098/rspb.2007. 0210

Shimizu, Y., and Kawasaki, T. (2021). Differential Regenerative Capacity of the Optic Tectum of Adult Medaka and Zebrafish. Front. Cell Dev. Biol. 9:686755. doi: 10.3389/fcell.2021.686755

Stenkamp, D. L. (2007). Neurogenesis in the Fish Retina. Int. Rev. Cytol. 259, 173-224. doi: 10.1016/s0074-7696(06)59005-9

Stenkamp, D. L. (2011). The rod photoreceptor lineage of teleost fish. Prog. Retin. Eye Res. 30, 395-404. doi: 10.1016/j.preteyeres.2011.06.004

Takaki, K., Ramakrishnan, L., and Basu, S. (2018). A zebrafish model for ocular tuberculosis. PLoS One 13:e0194982. doi: 10.1371/journal.pone.019 4982 
Tanaka, E. M., and Ferretti, P. (2009). Considering the evolution of regeneration in the central nervous system. Nat. Rev. Neurosci. 10, 713-723. doi: 10.1038/ nrn2707

Tsonis, P. A., and Del Rio-Tsonis, K. (2004). Lens and retina regeneration: transdifferentiation, stem cells and clinical applications. Exp. Eye Res. 78, 161-172. doi: 10.1016/j.exer.2003.10.022

Tuncdemir, S. N., Lacefield, C. O., and Hen, R. (2019). Contributions of adult neurogenesis to dentate gyrus network activity and computations. Behav. Brain Res. 374:112112. doi: 10.1016/j.bbr.2019.112112

Wan, J., and Goldman, D. (2016). Retina regeneration in zebrafish. Curr. Opin. Genet. Dev. 40, 41-47.

White, D. T., Sengupta, S., Saxena, M. T., Xu, Q., Hanes, J., Ding, D., et al. (2017). Immunomodulation-accelerated neuronal regeneration following selective rod photoreceptor cell ablation in the zebrafish retina. Proc. Natl. Acad. Sci. U.S.A. 114, E3719-E3728. doi: 10.1073/pnas.1617721114

Yun, M. H. (2015). Changes in Regenerative Capacity through Lifespan. Int. J. Mol. Sci. 16, 25392-25432. doi: 10.3390/ijms161025392
Conflict of Interest: The author declares that the research was conducted in the absence of any commercial or financial relationships that could be construed as a potential conflict of interest.

Publisher's Note: All claims expressed in this article are solely those of the authors and do not necessarily represent those of their affiliated organizations, or those of the publisher, the editors and the reviewers. Any product that may be evaluated in this article, or claim that may be made by its manufacturer, is not guaranteed or endorsed by the publisher.

Copyright (c) 2022 Blackshaw. This is an open-access article distributed under the terms of the Creative Commons Attribution License (CC BY). The use, distribution or reproduction in other forums is permitted, provided the original author(s) and the copyright owner(s) are credited and that the original publication in this journal is cited, in accordance with accepted academic practice. No use, distribution or reproduction is permitted which does not comply with these terms. 